\title{
Parallel and serial reduction pathways in complex oxide lithium-ion battery anodes
}

\author{
K. J. Griffith, K. R. Poeppelmeier \\ Northwestern University, 2145 Sheridan Road, Evanston, IL 60202 United States \\ kent.griffith@northwestern.edu
}

Complex early transition metal oxides have emerged as leading candidates for fast charging lithium-ion battery anode materials [1,2]. Framework crystal structures with frustrated topologies are good electrode candidates because they may intercalate large quantities of guest ions with minimal structural response. Starting from the empty perovskite $\left(\mathrm{ReO}_{3}\right)$ framework, shear planes and filled pentagonal columns are examples of motifs that decrease the structural degrees of freedom. As a consequence, many early transition metal oxide shear and bronze structures do not readily undergo the tilts and distortions that lead to phase transitions and/or the clamping of lithium diffusion pathways that occur in a purely corner-shared polyhedral network [1].

In this work, we explore the relationship between composition, crystal structure, and reduction pathway in a variety of recently synthesized mixed alkali, transition metal, and main group oxides (Fig. 1), moving beyond the archetypal Ti-Nb-O and W-Nb-O phase spaces. Solid-state NMR spectroscopy, X-ray absorption spectroscopy (XANES and EXAFS), synchrotron and neutron diffraction, and DFT are combined with electrochemical experiments to present a comprehensive picture of the charge storage mechanisms. Prospects for tunability and implications for charge rate and structural stability will be discussed.

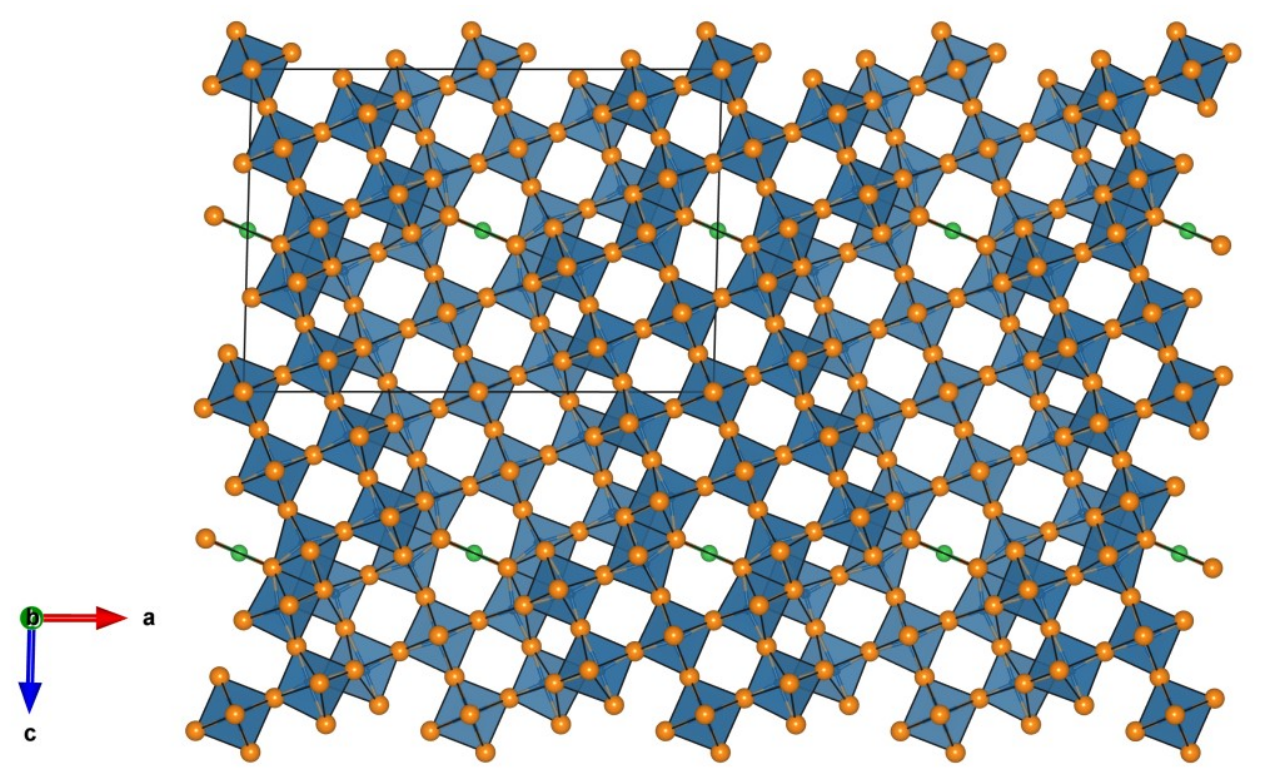

Figure 1. Crystal structure of the Wadsley-Roth crystallographic shear phase $\mathrm{NaNb}_{13} \mathrm{O}_{33}$.

[1] Griffith, K. J., Wiaderek, K., Cibin, G., Marbella, L. M. Grey, C. P. (2018). Nature 559, 556.

[2] Griffith, K. J., Harada, Y., Egusa, S., Ribas, R. M., Monteiro, R. S., Von Dreele, R. B., Cheetham, A. K., Cava, R. J., Grey, C. P., Goodenough, J. B. (2021). Chem. Mater. 33, 4.

Keywords: shear structures; Wadsley-Roth; X-ray absorption spectroscopy; solid-state NMR, neutron diffraction 